\title{
ADESÃO À VACINA CONTRA INFLUENZA POR PROFISSIONAIS E ESTUDANTES DE SAÚDE: UMA REVISÃO INTEGRATIVA
}

\author{
ADHERENCE TO INFLUENZA VACCINE BY PROFESSIONALS AND HEALTH \\ STUDENTS: AN INTEGRATIVE REVIEW
}

\section{Maridalva de Souza Penteado, Lacita Menezes Skalinski, Flávia Azevedo de Mattos Moura Costa, Adélia Maria Carvalho de Melo Pinheiro}

\begin{abstract}
To identify influenza vaccine adherence rates among health professionals and students and to verify which factors contribute to the increase or decrease of adherence to the immunobiological. The methodology used was the integrative review, searching the base of the Virtual Health Library. We analyzed 58 articles. There was variation in the rates, with some confluence in the results by country, with differences when comparing the professional categories. The belief in self-protection and the free availability of the vaccine contributed to adherence, while the belief that professionals are not a risk group, objection to the vaccine and its adverse events, lack of time and costs contributed to nonadherence. The organizational structure of the institutions seems to have relevance in adherence. However, part of the work focuses on the personal motives that lead the individual to join. Complementarily, other scientific investigations are suggested, with emphasis on institutions and their mechanisms for encouraging vaccination.
\end{abstract}

Key words: Vaccination Coverage; Influenza Vaccines; Health Personnel; Students.

\begin{abstract}
Resumo
Identificar as taxas de adesão à vacina contra influenza entre profissionais e estudantes da saúde e verificar que fatores concorrem para o aumento ou diminuição da aderência ao imunobiológico. Utilizou-se como metodologia a revisão integrativa, com busca na base da Biblioteca Virtual de Saúde. Foram analisados 58 artigos. Verificou-se variação nas taxas, havendo certa confluência nos resultados por país, com diferenças quando comparadas as categorias profissionais. A crença na autoproteção e a disponibilidade gratuita da vacina contribuíram para adesão, enquanto a crença de que os profissionais não se constituem em grupo de risco, objeção à vacina e seus eventos adversos, falta de tempo e custos colaboraram para a não adesão. A estrutura organizacional das instituições parece ter relevância na adesão. Contudo, parte dos trabalhos centra-se nos motivos pessoais que levam o indivíduo a aderir. Sugere-se, complementarmente, outras investigações científicas, com ênfase nas instituições e seus mecanismos de incentivo à vacinação.
\end{abstract}

Palavras Chave: Cobertura vacinal. Vacinas contra Influenza. Pessoal de Saúde. Estudantes. 
Introdução

A influenza, ou gripe, é uma enfermidade de alta transmissibilidade e difusão, cuja disseminação nos hospitais é bem documentada. Os relatos de epidemias de anos não pandêmicos fornecem evidências de infecção cruzada de paciente a paciente e da equipe para o paciente e vice-versa. $\mathrm{O}$ acometimento de profissionais da área da saúde pela influenza sempre se constituiu, portanto, em um risco conhecido ${ }^{1}$.

Intensificam-se, contudo, principalmente em períodos mais recentes, as preocupações e alertas de saúde pública de interesse internacional sobre o assunto, considerando o potencial do vírus de produzir pandemias, a exemplo do que foi vivenciado, em esfera global, nas epidemias de influenza $A$ (H5N1) (gripe aviária de 2004) e influenza A (H1N1) (gripe suína de 2009) ${ }^{2}$.

A pandemia de 2009, particularmente, sublinhou um problema antigo, o papel dos trabalhadores de saúde na transmissão da influenza, uma vez que os membros da equipe de saúde ocupam posição de relevo na propagação do vírus nos ambientes de saúde, sobretudo, nos hospitais ${ }^{3}$.

Estima-se que 13 a $20 \%$ dos trabalhadores da saúde não vacinados são infectados anualmente pelo vírus. Como muitos deles têm sintomas leves e moderados, normalmente continuam a trabalhar, contaminando pacientes e outros trabalhadores. Cinquenta por cento das infecções pela influenza são subclínicas e a transmissão do vírus tem início um dia antes da manifestação dos sintomas, o que facilita ainda mais sua disseminação $0^{4,5}$.

Como o pessoal da saúde apresenta risco especial de aquisição da doença, sua vacinação anual é necessária para reduzir o risco de adquirir a doença e evitar sua transmissão aos pacientes mais vulneráveis, com repercussões muitas vezes devastadoras ${ }^{3}$. Estudos revelam que a vacinação dos trabalhadores de saúde reduz a morbidade e a mortalidade de pacientes pela gripe, diminuindo, também, o inerente absenteísmo ao trabalho provocado pela doença, assim como a contaminação de outros profissionais. Mencionese que a própria falta ao trabalho gera problemas relevantes como os custos adicionais ao serviço e a sobrecarga das equipes desfalcadas, com consequências na assistência aos pacientes ${ }^{6}$.

A imunização contra a gripe se constitui, então, em elemento fundamental nos programas de prevenção e controle das infecções associadas aos cuidados em saúde e é fortemente recomendada para esse grupo por importantes órgãos como a OMS (Organização Mundial de Saúde), o CDC (Center for Disease Control), nos Estados Unidos, o ECDC (European Centre for Disease Prevention and Control), organização europeia de prevenção e controle de doenças, a Associação Pan-americana de Infectologia, dentre outros ${ }^{7,10}$.

Refere-se, todavia, grande variação nas taxas de vacinação dos profissionais de saúde para o vírus, nos diversos países e regiões, e, apesar de todos os esforços dos agentes envolvidos, as taxas mostram-se quase sempre menores que $50 \%$. Pelo exposto, realizou-se uma revisão integrativa da literatura, com o objetivo de identificar as taxas de vacinação contra a influenza entre profissionais e estudantes da área de saúde e, ainda, apontar os fatores que influenciam e dificultam a adesão a essa vacina por parte desses mesmos profissionais e discentes.

\section{Metodologia}

Trata-se de uma revisão integrativa da literatura, cujo propósito é reunir o conhecimento produzido sobre um tema, identificar, avaliar e sintetizar os resultados de pesquisas em uma área particular.

Para a busca dos artigos científicos, foi utilizada a base de dados da Biblioteca Virtual em Saúde (BVS). Visando à localização das publicações pertinentes ao estudo aqui proposto, foram utilizadas as palavras-chave: influenza, vaccination, healthcare workers.

Para a inclusão no estudo, foram utilizados os critérios: estudos que identificassem taxas de adesão à vacinação contra a influenza por parte de profissionais e estudantes da área de saúde, publicados no período de 2012 a 2016, nos idiomas português, inglês ou espanhol.

Foram excluídos trabalhos de revisão, editoriais e outros que não apontavam explicitamente taxas de vacinação de profissionais da saúde ou de estudantes da área contra o vírus da influenza e, ainda, aqueles que não estavam disponibilizados na íntegra.

Inicialmente, foram identificadas 507 publicações a partir das palavras-chave empregadas. Procedeu-se, então, à leitura dos títulos e resumos das pesquisas localizadas $a$ priori, e 58 estudos foram elegíveis por enquadrarem-se aos critérios pré-estabelecidos.

Numa próxima etapa, foi realizada a leitura dos escritos e preenchido um instrumento para a coleta dos seguintes dados: ano de publicação, 
idioma, país onde o estudo foi realizado, periódico no qual o estudo foi publicado, metodologia empregada, as taxas de vacinação e, caso fossem indicados, elementos preditivos e ou fatores que contribuíram para aumentar ou diminuir a adesão à vacinação antigripal.

\section{Resultados e Discussões}

Foram encontrados 58 artigos que objetivaram identificar taxas de adesão à vacinação contra a influenza de profissionais de saúde e estudantes, publicados entre 2012 e 2016, bem como os países nos quais foram realizados (Quadro 1).

Quadro 1. Artigos publicados e incluídos no estudo, com respectivo ano, periódico de publicação e país de realização da pesquisa.

\begin{tabular}{|c|c|c|c|c|}
\hline Artigo & Título & Ano & Periódico & País \\
\hline 1 & $\begin{array}{l}\text { Attitudes of dental healthcare workers towards the } \\
\text { influenza vaccination }^{11}\end{array}$ & 2012 & $\begin{array}{l}\text { Int J of Hyg and } \\
\text { Environmental } \\
\text { Health }\end{array}$ & Alemanha \\
\hline 2 & $\begin{array}{l}\text { Influenza vaccination coverage among patients and } \\
\text { healthcare workers in university hospital during } \\
\text { the } 2006-2007 \text { influenza season }{ }^{12}\end{array}$ & 2012 & Vaccine & França \\
\hline 3 & $\begin{array}{l}\text { Factors influencing uptake of influenza A (H1N1) } \\
\text { vaccine amongst healthcare workers in a regional } \\
\text { pediatric center: Lessons for improving vaccination } \\
\text { rates }^{13}\end{array}$ & 2012 & Vaccine & Escócia \\
\hline 4 & $\begin{array}{l}\text { Factors Affecting Medical Students' Uptake of the } \\
2009 \text { Pandemic Influenza A (H1N1) }\end{array}$ & 2012 & $\begin{array}{l}\text { Influenza Res } \\
\text { Treat }\end{array}$ & Inglaterra \\
\hline 5 & $\begin{array}{l}\text { Personal decision-making criteria related to } \\
\text { seasonal and pandemic } A(H 1 N 1) \text { influenza- } \\
\text { vaccination acceptance among French healthcare } \\
\text { workers }^{15}\end{array}$ & 2012 & Plos One & França \\
\hline 6 & $\begin{array}{l}\text { Barriers to pandemic influenza vaccination and } \\
\text { uptake of seasonal influenza vaccine in the post- } \\
\text { pandemic season in Germany }{ }^{16}\end{array}$ & 2012 & BMC Public Health & Alemanha \\
\hline 7 & $\begin{array}{l}\text { A public health initiative to increase annual } \\
\text { influenza immunization among }{ }^{17} \\
\text { hospital health care personnel: The San Diego } \\
\text { Hospital influenza immunization } \\
\text { partnership }{ }^{17}\end{array}$ & 2012 & $\begin{array}{l}\text { American Journal } \\
\text { of Infection } \\
\text { Control }\end{array}$ & $\begin{array}{l}\text { Estados } \\
\text { Unidos }\end{array}$ \\
\hline 8 & $\begin{array}{l}\text { Changes in influenza vaccination rates among } \\
\text { healthcare workers following a pandemic influenza } \\
\text { year at a Japanese tertiary care center }{ }^{18}\end{array}$ & 2012 & $\begin{array}{l}\text { Journal of Hospital } \\
\text { Infection }\end{array}$ & Japão \\
\hline 9 & $\begin{array}{l}\text { H1N1 and seasonal influenza vaccination of } \\
\text { U.S. healthcare personnel, } 2010^{19}\end{array}$ & 2012 & Am J Prev Med & $\begin{array}{l}\text { Estados } \\
\text { Unidos }\end{array}$ \\
\hline 10 & $\begin{array}{l}\text { High effectiveness of pandemic influenza } A(H 1 N 1) \\
\text { vaccination in healthcare workers from a } \\
\text { Portuguese hospital }{ }^{20}\end{array}$ & 2012 & $\begin{array}{l}\text { Int Arch Occup } \\
\text { Environ Health }\end{array}$ & Portugal \\
\hline 11 & $\begin{array}{l}\text { Influenza vaccination among healthcare personnel } \\
\text { after pandemic influenza } \mathrm{H} 1 \mathrm{~N} 1^{21}\end{array}$ & 2012 & Vaccine & Espanha \\
\hline 12 & $\begin{array}{l}\text { Influenza vaccination coverage among health-care } \\
\text { personnel }-2011-12 \text { influenza season, United } \\
\text { States }^{22}\end{array}$ & 2012 & $\begin{array}{l}\text { MMWR Morb } \\
\text { Wkly Report }\end{array}$ & $\begin{array}{l}\text { Estados } \\
\text { Unidos }\end{array}$ \\
\hline 13 & $\begin{array}{l}\text { Seasonal and H1N1 influenza vaccine compliance } \\
\text { and intent to be vaccinated } \\
\text { among emergency medical services personnel }{ }^{23}\end{array}$ & 2012 & $\begin{array}{l}\text { American Journal } \\
\text { of Infection } \\
\text { Control }\end{array}$ & $\begin{array}{l}\text { Estados } \\
\text { Unidos }\end{array}$ \\
\hline 14 & $\begin{array}{l}\text { Actitudes, percepciones y factores asociados a la } \\
\text { vacunación antigripal en los profesionales de } \\
\text { atención primaria de Navarra, 2011-2012 }\end{array}$ & 2013 & An.Sist Navar & Espanha \\
\hline
\end{tabular}


...continuação

15 Seasonal influenza vaccination coverage rate of target groups in select cities and provinces in China by season (2009/10 to $2011 / 12)^{25}$

16 Determinants of influenza vaccination uptake among Italian healthcare workers ${ }^{26}$

2013

Influenza and hepatitis B vaccination coverage among healthcare workers in Croatian hospitals: a series of cross-sectional surveys, 2006-2011 ${ }^{27}$

18 Knowledge of and attitudes to influenza vaccination in healthy primary healthcare workers in Spain, 2011-2012 28

19 Predictive factors associated with the acceptance of pandemic and seasonal influenza vaccination in healthcare workers and students in Tuscany, central Italy ${ }^{29}$

20 Evaluating influenza vaccination campaigns beyond coverage: A before-after study among health care workers $^{30}$

21 Influenza immunization coverage of residents and employees of long-term care facilities in New York State, $2000-2010^{31}$

Influenza vaccination among health care personnel in California: 2010-2011 influenza season ${ }^{32}$

23 Influenza vaccination coverage among health care personnel - United States, 2012-13 influenza season $^{33}$

24 Pandemic $A(H 1 N 1) 2009$ influenza vaccination in Lyon university hospitals, France: Perception and attitudes of hospital workers ${ }^{34}$

25 Predicting influenza vaccination uptake among health care workers: What are the key motivators? ${ }^{35}$

26 Prevalence and factors associated with 2009 to 2011 influenza vaccinations at a university medical center $^{36}$

27 Improvement in attitudes toward influenza vaccination in medical students following an integrated curricular intervention ${ }^{37}$

28 Qualitative motivators and barriers to pandemic vs seasonal influenza vaccination among health care workers: A content analysis ${ }^{38}$

29 Influenza immunization coverage for healthcare workers in a community hospital in Qatar (20112012 and 2013-2013 seasons) ${ }^{39}$

30 Association between health care workers' knowledge of influenza vaccine and vaccine uptake $^{40}$

31 Barriers and facilitators to influenza vaccination and vaccine coverage in a cohort of health care personnel ${ }^{41}$

32 Health care professionals' awareness of knowledge about and attitude to influenza vaccination $^{42}$

\begin{tabular}{|c|c|c|}
\hline 2013 & Pios ome & Crillid \\
\hline 2013 & $\begin{array}{l}\text { Hum Vaccin } \\
\text { Immunother }\end{array}$ & Itália \\
\hline 2013 & BMC Infect Dis & Croácia \\
\hline 2013 & Plos One & Espanha \\
\hline 2013 & $\begin{array}{l}\text { Hum Vaccin } \\
\text { immunother }\end{array}$ & Itália \\
\hline 2013 & $\begin{array}{c}\text { American Journal } \\
\text { of Infection } \\
\text { Control }\end{array}$ & Espanha \\
\hline 2013 & $\begin{array}{c}\text { American Journal } \\
\text { of Infection } \\
\text { Control }\end{array}$ & $\begin{array}{l}\text { Estados } \\
\text { Unidos }\end{array}$ \\
\hline 2013 & $\begin{array}{c}\text { American Journal } \\
\text { of Infection } \\
\text { Control }\end{array}$ & $\begin{array}{l}\text { Estados } \\
\text { Unidos }\end{array}$ \\
\hline 2013 & $\begin{array}{l}\text { MMWR Morb } \\
\text { Wkly Report }\end{array}$ & $\begin{array}{l}\text { Estados } \\
\text { Unidos }\end{array}$ \\
\hline 2013 & Vaccine & França \\
\hline 2013 & $\begin{array}{c}\text { American Journal } \\
\text { of Infection } \\
\text { Control }\end{array}$ & Canadá \\
\hline 2013 & $\begin{array}{c}\text { American Journal } \\
\text { of Infection } \\
\text { Control }\end{array}$ & $\begin{array}{l}\text { Estados } \\
\text { Unidos }\end{array}$ \\
\hline 2014 & Vaccine & $\begin{array}{l}\text { Estados } \\
\text { Unidos }\end{array}$ \\
\hline 2014 & Vaccine & Canadá \\
\hline 2014 & $\begin{array}{l}\text { Journal Of } \\
\text { Infection and } \\
\text { public health }\end{array}$ & Qatar \\
\hline 2014 & $\begin{array}{c}\text { American Journal } \\
\text { of Infection } \\
\text { Control }\end{array}$ & $\begin{array}{l}\text { Estados } \\
\text { Unidos }\end{array}$ \\
\hline 2014 & $\begin{array}{c}\text { American Journal } \\
\text { of Infection } \\
\text { Control }\end{array}$ & $\begin{array}{l}\text { Estados } \\
\text { Unidos }\end{array}$ \\
\hline 2014 & Vaccine & $\begin{array}{l}\text { Arábia } \\
\text { Saudita }\end{array}$ \\
\hline
\end{tabular}


...continuação

33 Influenza vaccination coverage among health care personnel - United States, 2013-14 influenza season $^{43}$

Knowledge, attitudes and behavior of hospital health-care workers regarding influenza A/H1N1: a cross sectional survey ${ }^{44}$

35 Vaccination coverage for seasonal influenza among residents and health care workers in Norwegian nursing homes during the $2012 / 13$ season, a crosssectional study ${ }^{45}$

36 Influenza vaccination rates and beliefs about vaccination among nursing home employees ${ }^{46}$

37 Vacunación antigripal en estudiantes de enfermería durante la temporada 2014-2015 ${ }^{47}$

Factores asociados a recibir la vacunación antigripal en profesionales de atención primaria ${ }^{48}$

39 Sociocognitive predictors of the intention of healthcare workers to receive the influenza vaccine in Belgian, Dutch and German hospital settings ${ }^{49}$

40 Visualizing knowledge and attitude factors related to influenza vaccination of physicians ${ }^{50}$

41 Promotion of influenza vaccination among healthcare workers: findings from a tertiary care children's hospital ${ }^{51}$

42 An effective strategy for influenza vaccination of healthcare workers in Australia: experience at a large health service without a mandatory policy ${ }^{52}$

43 A new approach to improving healthcare personnel influenza immunization programs: A 2015 randomized Controlled trial ${ }^{53}$

$44 \quad$ Factors affecting uptake of recommended immunizations among health care workers in South Australia ${ }^{54}$

45 Influenza vaccination among healthcare workers in Italy. The experience of a large tertiary acute-care teaching hospital ${ }^{55}$

46 Influenza vaccination coverage among health care personnel - United States, 2014-15 influenza season

47 Seasonal influenza vaccination amongst medical students: A social network analysis based on a cross-sectional Study ${ }^{56}$

48 Sustained low influenza vaccination in health care workers after H1N1 pandemic: a cross sectional study in an Italian healthcare setting for at-risk patients $^{57}$

49 Barriers of Influenza vaccination in healthcare personnel in France ${ }^{58}$

50 Could University training and proactive attitude of coworkers be associated with influenza vaccination compliance? A multicenter survey among Italian medical residents ${ }^{59}$

51 Knowledge, attitudes and practices of Australian medical students towards influenza vaccination ${ }^{60}$
2014

MMWR Morb

Wkly Report

Estados

unidos

2014

BMC Infect

Disease

Itália

2014

BMC Public Health

Noruega

2015

2015

2015

2015

Journal of Hospital Infection

Am Journal of

Estados Infect Control Unidos Rev Esp Salud Publica

Espanha

Gac Sanit

Espanha

Bélgica, Alemanha e Holanda

2015

Vaccine

Espanha

2015

BMC Public Health

Itália

2015

BMC Infectious

Diseases

Austrália

Plos One |

Canadá

Human Vaccines \& Immunotherapeut ics

Human Vaccines \&

2015

Immunotherapeut

ics

2015

MMWR Morb
Wkly Report

Estados

Unidos

2015

Plos One

Inglaterra

2015

BMC Infect Dis

Itália

Am Journal Infect Control

França

2016

BMC Med Educ

Itália

2016
Vaccine

Austrália 
...continuação

52 Factors effecting influenza vaccination uptake among healthcare workers: A multicenter cross sectional study ${ }^{61}$

53 Determinants of adherence to seasonal influenza vaccination among healthcare workers from Italian region: results from a cross-sectional study ${ }^{62}$

54 Adherence to influenza vaccination among medical students during and after Influenza A (H1N1) pandemic $^{63}$

55 Influenza vaccination among Malaysian healthcare workers: a survey of coverage and attitudes ${ }^{64}$

2016

BMC Infect Dis

Turquia

2016

BMJ Open

Itália

2016

Rev Inst. Med. Trop S Paulo

Brasil

2016

Med J Malaysia Malásia

56 Influenza vaccination coverage among health care personnel - United States, 2015-16 influenza season ${ }^{65}$

2016

MMWR Morb

Estados

Wkly Report Unidos

57 Influenza vaccination coverage rates, knowledge, attitudes, and beliefs in Jordan: A comprehensive study ${ }^{66}$

58 Promotion of flu vaccination among healthcare workers in an Italian academic hospital: An experience with tailored web tools ${ }^{67}$

2016 Viral Immunology Jordânia

Human Vaccines \& Immunotherapeut ics
Itália
Observaram-se variações expressivas nas taxas de vacinação, ainda que se considere as diversas categorias profissionais estudadas, bem como diferentes populações e amostras e, sobretudo, metodologias empregadas, com destaque para as distintas formas de coleta de dados. As taxas de adesão à vacinação, no geral, e segundo a categoria dos profissionais e estudantes, são apresentadas no Quadro 2.

Quadro 2. Taxas de adesão à vacina antigripal, por profissionais e estudantes da área de saúde, identificadas nos estudos publicados.

\begin{tabular}{|c|c|c|c|}
\hline Artigo & Categoria Profissional & Amostra & Taxa de Adesão à Vacinação \\
\hline 1 & $\begin{array}{l}\text { Dentistas e estudantes de odontologia } \\
\text { de um hospital universitário de } \\
\text { Frankfurt- Alemanha }\end{array}$ & $\begin{array}{l}174 \text { dentistas } \\
570 \text { estudantes } \\
\text { de odontologia }\end{array}$ & $31,6 \%-H 1 N 1$ (2009) \\
\hline 2 & $\begin{array}{l}\text { Médicos, enfermeiros residentes de } \\
\text { medicina, técnicos de enfermagem, } \\
\text { pessoal da nutrição e outros } \\
\text { profissionais administrativos de quatro } \\
\text { setores de hospital universitário } \\
\text { francês }\end{array}$ & 105 & $\begin{array}{c}28 \%(2006 / 7) \\
73 \% \text { - Médicos } \\
43 \% \text { - Residentes } \\
23 \% \text { - Enfermeiros } \\
15 \% \text { - Téc. de enfermagem } \\
17 \% \text { - Outras categorias }\end{array}$ \\
\hline 3 & $\begin{array}{l}\text { Profissionais da Saúde de hospital } \\
\text { regional de pediatria na Escócia }\end{array}$ & 260 & $\begin{array}{l}49,6 \%(2010) \\
\text { Maiores taxas encontradas entre } \\
\text { médicos e homens }\end{array}$ \\
\hline 4 & $\begin{array}{l}\text { Estudantes de } \quad \begin{array}{l}\text { medicina da } \\
\text { Universidade } \\
\text { Inglaterra }\end{array} \\
\text { In }\end{array}$ & 205 & $49,2 \%$ \\
\hline 5 & $\begin{array}{l}\text { Médicos e paramédicos de cinco } \\
\text { hospitais franceses, localizados em } \\
\text { Paris e nos subúrbios }\end{array}$ & 1.480 & $\begin{array}{l}\text { 30\% - paramédicos (sazonal) } \\
21 \% \text { - paramédicos (H1N1) } \\
58 \% \text { - médicos (sazonal) } \\
71 \% \text { - médicos (H1N1) }\end{array}$ \\
\hline 6 & $\begin{array}{l}\text { População adulta em geral e } \\
\text { profissionais da saúde da Alemanha }\end{array}$ & 2.493 & $\begin{array}{l}\text { 30,5\% -profissionais de saúde } \\
\text { 8,85\% - população adulta }\end{array}$ \\
\hline
\end{tabular}


Trabalhadores da saúde de quatorze hospitais localizados em San DiegoEstados Unidos

Trabalhadores da saúde em instituição

8 de atenção médica terciária em Sapporo-Japão

Profissionais da saúde e população em

9 geral, dos Estados Unidos

Profissionais da saúde do hospital do

10 Porto - Portugal

Trabalhadores da saúde de Alicante-

11 Espanha

Profissionais da saúde dos Estados Unidos

12

Trabalhadores da saúde de uma

13 unidade de emergência em Saint Louis, Estados Unidos

Médicos de família, pediatras, enfermeiros e técnicos de

14 enfermagem atuantes na atenção primária em sete comunidades autônomas da Espanha

Trabalhadores da saúde atuantes em

15 serviços de saúde de cinco províncias da China

16 Trabalhadores da Saúde da Itália
Dados de

registros

hospitalares

1.489

Dados de

registro

hospitalar

16.975

5.592

3.126

2.348

265

$73,6 \%(2010 / 11)$

$68,5 \%$ - H1N1

$60,9 \%(2008 / 9)$

61,2\% (2009/10)

$58,4 \%(2010 / 11)$

$56,2 \%(2011 / 12)$

573

$9,5 \%$

5.336

$20 \%$ 


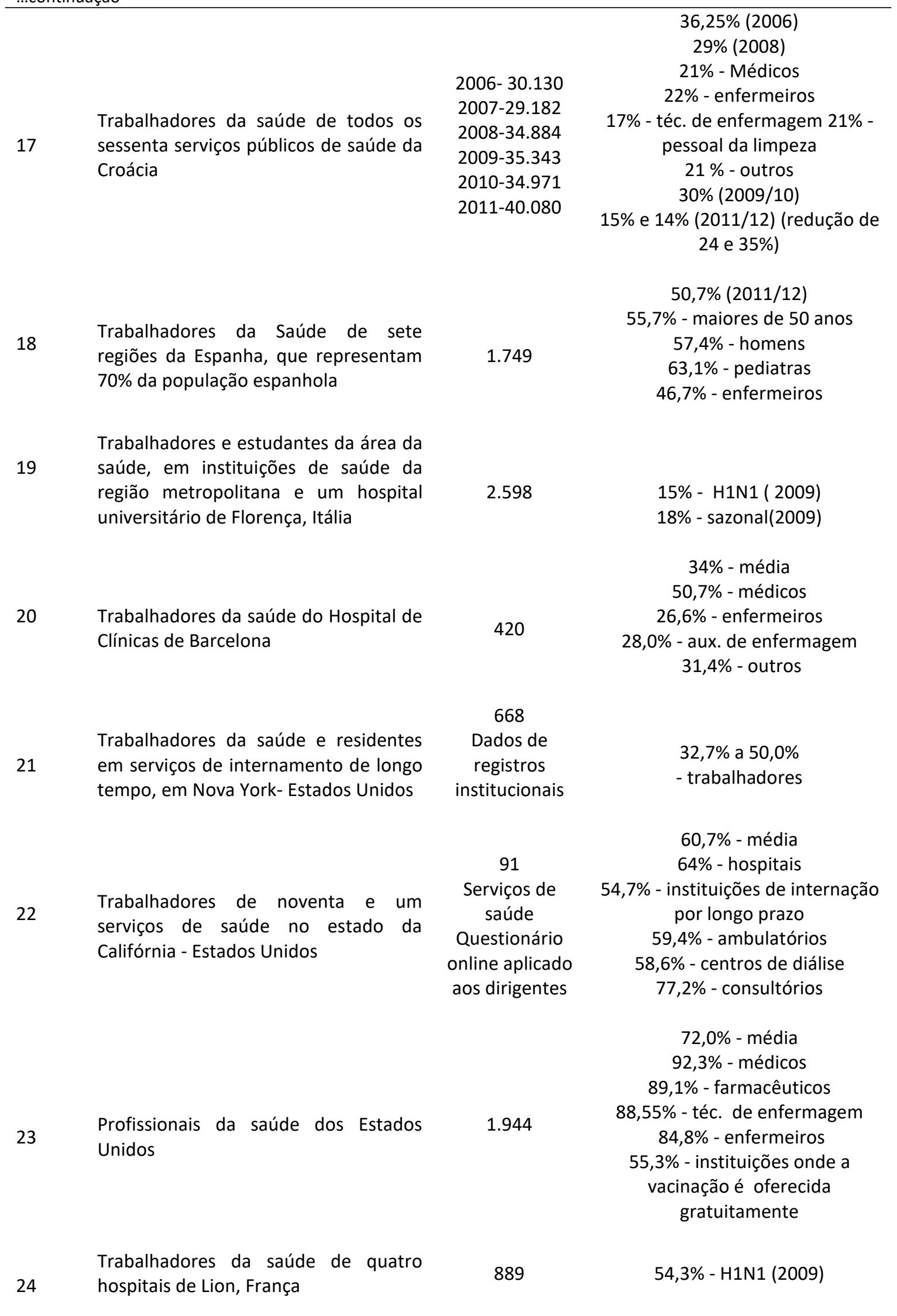


25 Profissionais da saúde em um hospital de grande porte no Canadá

3.275

Trabalhadores da área clínica e não clínica de um centro médico universitário nos Estados Unidos

Estudantes de medicina da Oakland

27 University, no Michigan, Estados Unidos

Médicos, enfermeiros, laboratoristas, pessoal da administração, da limpeza e de apoio de um hospital de atenção terciária em Ontario, Canadá

Médicos, enfermeiros e ocupacionais de enfermagem atuantes em hospital de setenta e cinco leitos, no Qatar

Profissionais da saúde do Hospital

30 Universitário da Carolina do SulEstados Unidos

31 Profissionais da saúde do TexasEstados Unidos

Profissionais da saúde dos seis maiores hospitais da Arábia Saudita

Profissionais da Saúde dos Estados Unidos hospitais universitários em Nápoles e Avelino na Itália

Residentes (Pacientes) e trabalhadores de 354 casas de repouso na Noruega
1.882

600

Dados de

registros nas campanhas de

2011/12;

2012/13

689

1.782

residentes

28.237
$87,4 \%-\mathrm{H} 1 \mathrm{~N} 1$

71\% - área clínica (H1N1)

$27 \%$ - área não clínica (H1N1)

$82 \%$ - área clínica (2009/10)

$42 \%$ - área não clínica (2009/10)

$73 \%$ - área clínica (2010/11)

28\% - área não clínica (2010/11)

$48 \%$

$87,4 \%$ - H1N1

$74,3 \%$ - sazonal

$61,7 \%$ - média (2011/12)

$69,3 \%$ enfermeiros(2011/12)

46,9\% - médicos (2011/12)

$65,9 \%$ - técnicos (2011/12)

$71,1 \%$ - média (2012/13)

$73,9 \%$ enfermeiros(2012/13)

$69,2 \%$ - médicos (2012/13)

$66,2 \%$ - técnicos (2012/13)

$73 \%(2009 / 10)$

$94 \%(2010 / 11)$

$77 \%(2010 / 11)$

$38 \%$

$75,2 \%$ - média (2013/14)

$92,2 \%$ - médicos

$90,5 \%$ - enfermeiros

$89,6 \%$ - téc. de enfermagem $57,7 \%$ - assistentes e cuidadores $68,6 \%$ - outras ocupações não clínicas

$$
17,7 \%
$$

$71,7 \%$ - residentes $0 \%$ - trabalhadores 


\begin{tabular}{|c|c|c|c|}
\hline 36 & $\begin{array}{l}\text { Enfermeiros atuantes em casas de } \\
\text { repouso (Trinta e sete serviços), na } \\
\text { Flórida, Wisconsin e Geórgia }\end{array}$ & 1.965 & $54 \%(2011 / 12)$ \\
\hline 37 & $\begin{array}{l}\text { Estudantes de enfermagem da } \\
\text { Universidade de Salamanca, Espanha }\end{array}$ & 340 & $5,3 \%(2014 / 15)$ \\
\hline 38 & $\begin{array}{l}\text { Médicos da família, pediatras } \\
\text { enfermeiros, na região sanitária de } \\
\text { Lleida, Espanha }\end{array}$ & 287 & $60,3 \%(2013 / 14)$ \\
\hline 39 & $\begin{array}{l}\text { Enfermeiros, médicos, paramédicos } \\
\text { fisioterapeutas e outros } \\
\text { administrativos de hospitais da } \\
\text { Alemanha, Bélgica e Holanda }\end{array}$ & 1.022 & $37,2 \%(2012 / 13)$ \\
\hline 40 & $\begin{array}{l}\text { Médicos atuantes na atenção primária } \\
\text { em sete regiões da Espanha - } \\
\text { Andalusia, Castile- Leon, Catalônia, } \\
\text { Valença, Navarra e País Basco }\end{array}$ & 1.749 & $50,7 \%$ \\
\hline 41 & $\begin{array}{l}\text { Trabalhadores da saúde de hospital } \\
\text { universitário, pediátrico, de atenção } \\
\text { terciária na Itália }\end{array}$ & 191 & $\begin{array}{l}\text { 35,6\% - vacinados pelo menos } \\
\text { uma vez } \\
\text { 6,8\% - referiram vacinação anual }\end{array}$ \\
\hline 42 & $\begin{array}{l}\text { Trabalhadores da saúde de hospital } \\
\text { universitário na Austrália }\end{array}$ & 1.328 & $\begin{array}{c}76 \% \text { - inicialmente } \\
80,0 \% \text { - depois de campanha }\end{array}$ \\
\hline & & & $\begin{array}{c}43 \%(2009 / 10) \\
44 \%(2011)\end{array}$ \\
\hline 43 & $\begin{array}{l}\text { Profissionais de saúde de quarenta e } \\
\text { três instituições no Canadá }\end{array}$ & $\begin{array}{l}\text { Dados obtidos } \\
\text { através da } \\
\text { informação dos } \\
\text { dirigentes }\end{array}$ & $\begin{array}{l}55 \% 2011) \\
\text { Grupo controle: } \\
62 \%(2009 / 10) \\
57 \%(2011) \\
55 \%(2012)\end{array}$ \\
\hline 44 & Trabalhadores da saúde - Austrália & 92 & $80 \%$ \\
\hline 45 & $\begin{array}{l}\text { Profissionais da saúde do Hospital San } \\
\text { Martin - Gênova - Itália }\end{array}$ & $\begin{array}{l}\text { Dados de } \\
\text { registro } \\
\text { hospitalar }\end{array}$ & $\begin{array}{l}56 \%(2005 / 06) \\
20 \%(2006 / 07) \\
24 \%(2007 / 08) \\
30 \%(2008 / 09) \\
34 \%(2009 / 10) \\
20 \%(2010 / 11) \\
18 \%(2011 / 12) \\
11 \%(2012 / 13) \\
16 \%(2013 / 14)\end{array}$ \\
\hline
\end{tabular}




\begin{tabular}{|c|c|c|c|}
\hline 46 & $\begin{array}{l}\text { Profissionais da saúde dos Estados } \\
\text { Unidos }\end{array}$ & 1.914 & $\begin{array}{c}77,3 \% \text { - média } \\
95,3 \% \text { - farmacêuticos } \\
64,4 \% \text { - assistentes e cuidadores } \\
96,0 \% \text { - instituições onde a } \\
\text { vacinação é obrigatória } \\
\text { 83,9\% - onde não é obrigatória e } \\
\text { ocorre vacinação sem custo por } \\
\text { vários dias } \\
73,6 \% \text { - quando ocorre em } \\
\text { somente um dia }\end{array}$ \\
\hline 47 & $\begin{array}{l}\text { Estudantes de medicina da } \\
\text { universidade de Lancaster- Inglaterra }\end{array}$ & 217 & $65 \%$ \\
\hline 48 & $\begin{array}{l}\text { Profissionais da saúde de hospital } \\
\text { universitário localizado no sudeste da } \\
\text { Itália }\end{array}$ & 400 & $\begin{array}{l}17 \% \text { - sazonal } \\
37 \% \text { - H1N1 }\end{array}$ \\
\hline 49 & $\begin{array}{l}\text { Médicos, enfermeiros e técnicos de } \\
\text { enfermagem de sessenta e sete } \\
\text { instituições de saúde da França }\end{array}$ & 3.213 & $22,3 \%(2012 / 13)$ \\
\hline 50 & $\begin{array}{l}\text { Médicos residentes de dezoito } \\
\text { universidades italianas }\end{array}$ & 2.506 & $11,9 \%(2011 / 12)$ \\
\hline 51 & $\begin{array}{l}\text { Estudantes de medicina de um } \\
\text { hospital universitário de grande porte } \\
\text { na Austrália }\end{array}$ & 606 & $53,8 \%(2014)$ \\
\hline 52 & $\begin{array}{l}\text { Médicos e enfermeiros de cinco } \\
\text { hospitais universitários localizados no } \\
\text { sudeste da Turquia }\end{array}$ & 642 & $\begin{array}{c}\text { 9,2\% - média } \\
15,2 \% \text { - médicos } \\
8,2 \% \text { - enfermeiros }\end{array}$ \\
\hline 53 & $\begin{array}{l}\text { Trabalhadores da saúde de dois } \\
\text { serviços de saúde, localizados em } \\
\text { Ligúria, nordeste da Itália }\end{array}$ & 830 & $\begin{array}{c}26,4 \%(2013 / 14) \\
12,5 \% \text { (Últimas } 6 \text { estações) } \\
48,4 \% \text { (Nunca foi vacinado } \\
\text { durante o período) }\end{array}$ \\
\hline 54 & $\begin{array}{l}\text { Estudantes de medicina de } \\
\text { universidade pública, localizada em } \\
\text { São Paulo- Brasil }\end{array}$ & 144 & $\begin{array}{c}91 \%-\mathrm{H} 1 \mathrm{~N} 1(2010) \\
42 \%(2011)\end{array}$ \\
\hline 55 & $\begin{array}{l}\text { Profissionais da saúde de hospital na } \\
\text { Malásia }\end{array}$ & 527 & média - 51,4\% (2013) \\
\hline 56 & $\begin{array}{l}\text { Profissionais da saúde dos Estados } \\
\text { Unidos }\end{array}$ & 2.258 & $\begin{array}{c}79 \% \text { - média } \\
91,2 \% \text { - trabalhadores } \\
\text { hospitalares } \\
79,8 \% \text { - ambulatórios } \\
69,2 \% \text { - serviços de internamento } \\
\text { de longo tempo }\end{array}$ \\
\hline 57 & $\begin{array}{l}\text { População em geral e profissionais da } \\
\text { saúde na Jordânia }\end{array}$ & 522 & $\begin{array}{c}36 \% \text { - média } \\
18,2 \%(2011 / 12) \\
33,50 \%(2008-12)\end{array}$ \\
\hline 58 & $\begin{array}{l}\text { Profissionais da saúde de hospital de } \\
\text { grande porte localizado no nordeste } \\
\text { da Itália }\end{array}$ & 501 & $\begin{array}{l}7,6 \%(2013 / 14) \\
5,6 \%(2012 / 13)\end{array}$ \\
\hline
\end{tabular}


Como motivos que favorecem a vacinação destacam-se a crença dos profissionais de estarem se auto protegendo e também aos seus familiares e, por fim, mas com menor destaque, a proteção aos pacientes. É digno de relevo o crédito à efetividade da vacina, sua disponibilidade nos serviços, o correto entendimento de que o profissional de saúde se encontra mais exposto à infecção e a existência de protocolos institucionais que determinem a necessidade da vacinação (Quadro 3).

Quadro 3. Fatores que contribuíram para aumentar a adesão à vacinação contra a gripe, de acordo com os resultados explicitados nos estudos analisados.

\begin{tabular}{|c|c|}
\hline Artigo & Fatores que contribuíram para aumentar a adesão \\
\hline 1 & $\begin{array}{l}\text { Autoproteção - 96,2\%; proteção dos pacientes - 59,2\%; proteção de amigos e familiares - } \\
58,6 \%\end{array}$ \\
\hline 3 & Alto risco de contato com H1N1 (88\%); responsabilidade de proteger os pacientes - $71 \%$ \\
\hline 4 & $\begin{array}{l}\text { Reduzir a chance de infecção por H1N1 - 82,2\%; reduzir a chance de transmissão do H1N1 } \\
\text { para os pacientes - } 75,9 \% \text {; reduzir a chance de transmissão do H1N1 para os familiares - } 63,8 \%\end{array}$ \\
\hline 5 & Ser mais velho; trabalhar em emergência ou terapia intensiva; ser médico \\
\hline 6 & $\begin{array}{l}\text { Ser médico; acreditar que a vacina é segura; crer que os trabalhadores da saúde têm maiores } \\
\text { riscos de ter gripe; estar ciente de que os trabalhadores da saúde devem receber a vacina } \\
\text { anualmente }\end{array}$ \\
\hline 9 & Ser homem; ter maior escolaridade; ser casado; nunca ter fumado; ter plano de saúde \\
\hline & Ser homem; ter mais de 45 anos \\
\hline 11 & $\begin{array}{l}\text { Autoproteção (67\%-70,6\%); vacinação prévia; proteger a família } 49,1 \%-57,7 \%) \text {; proteger os } \\
\text { pacientes }(49,4-56,3 \%)\end{array}$ \\
\hline
\end{tabular}

13 Trabalhar em instituições onde a vacinação é obrigatória

Crença de que o pessoal de emergência tem que se vacinar todo ano; importância da vacinação; percepção de que a vacina tem poucos eventos adversos; vacinação prévia

14 Conhecimento de que a vacina é efetiva; preocupação em ficar doente por infectar-se no trabalho; preocupação em contaminar pacientes e familiares

Ser homem; ter mais de 44 anos; ser portadores de diabetes e de doenças cardíacas; ter nível educacional mais alto

18 Considerar que a vacinação do profissional de saúde é importante; considerar que a vacina é efetiva

Ser homem; ter pós-graduação; ser médico; ser ocupante de cargo de direção; ser profissional de manutenção ou técnico não médico; ser trabalhador de hospital universitário

24 Proteger os familiares - 82,4\%; autoproteção - 65,8\%; proteger os pacientes - 57,1\%; ser homem; ter mais de 50 anos; trabalhar em terapia intensiva; ginecologia e pediatria

25 Ser mais velho; ter relacionamento estável; ter dependentes e filho; ter convívio familiar com crianças, pessoas idosas e com doenças crônicas; ter vacinação prévia; trabalhar em período integral; percepção de que tinham maior risco de adquirir H1N1 do que a população em geral; percepção de que a vacina protege também os pacientes; percepção de que o H1N1 é uma doença severa; percepção de que todos os trabalhadores precisam ser vacinados 
28 Motivações pessoais; prevenir infecções em pessoas de sua relação(familiares, pacientes e comunidade)

29 Ser enfermeiro(a), quando comparado aos médicos e técnicos de enfermagem

30 Obrigatoriedade de assinar termo de responsabilidade por não se vacinar (33\%); autoproteção - 28\%; proteção dos pacientes - $26 \%$

31 Ser mais velho (entre 50 e 65 anos); estar casado ou ter parceiro; ser médico ou dentista; ter vacinação prévia; ter 21 ou mais anos de trabalho, ter alta satisfação com o trabalho

36 Ser enfermeira; ser branca; ser mais velha; trabalhar em instituição que dispõe de dispositivos de incentivo à vacinação

37 Autoproteção - 75\%; proteção da família e amigos - 58,3\%; proteção dos pacientes - 50,0\%

38 Percepção de que a vacina protege a própria saúde; crença na efetividade da vacina

39 Ser belga ou holandês quando comparado aos alemães; percepção de estar mais suscetível à doença; vacinação prévia

41 Proteção dos pacientes - 34,3\%

42

Ter a vacina à disposição; comunicação; marketing

44 Autoproteção; proteger os pacientes; ser mais jovem

48 Entender que pertence a um grupo de risco para a infecção

50 Ter conhecimento correto sobre a vacina; ter lido artigos científicos sobre o imunobiológico

51 Morar no campus universitário; ter experiência clínica; concordar que a vacina é importante

52 Ser homem; ser médico; ter maior tempo de serviço; trabalhar em clínica médica; ter doença crônica; conviver com pessoa com mais de 65 anos de idade

53 Ser médico; concordar que a vacina é segura; acreditar que trabalhadores da saúde têm mais risco de adquirir gripe e estar convencido de que o trabalhador da saúde deva receber vacina anualmente; proteção da família - 53,9\%; prevenção da gripe - 53,4\%; proteção dos pacientes $-35,2 \%$ Autoproteção; facilidade de se obter a vacina

Como razões que afastam os profissionais da vacinação, os trabalhos destacam o desconhecimento sobre a vacina, a crença de que os profissionais da saúde não se constituem em grupo de risco, a objeção à vacina e a seus supostos eventos adversos. Adicionalmente, há percepção de haver pressões da indústria farmacêutica para seu emprego para além do fundamentalmente indicado e, ainda, o baixo acesso à vacina, a falta de tempo e os custos da vacina quando ela não é ofertada gratuitamente pelos serviços (Quadro 4).

Quadro 4. Fatores que contribuíram para diminuir a adesão à vacinação contra a gripe, por profissionais e estudantes da área de saúde, de acordo com os resultados explicitados nos estudos analisados.

\begin{tabular}{ll}
\hline Artigo & \multicolumn{1}{c}{ Fatores que contribuíram para diminuir a adesão } \\
\hline 1 & $\begin{array}{l}\text { Objeção à vacina - 61,1\%; preocupação com os eventos adversos } \\
\text { Incerteza sobre os eventos adversos - 47\%; incerteza sobre a segurança da vacina - 33\%; estar } \\
\text { muito ocupado - 22\% }\end{array}$ \\
$\begin{array}{l}\text { Supostos eventos adversos da vacina - 67,9\%; falta de informação sobre a vacina - 50,9\%; } \\
\text { percepção de não estar sob risco de ter gripe }\end{array}$ \\
\hline
\end{tabular}


6 A indústria farmacêutica influencia as decisões sobre estratégias de vacinação

7 Crença de que não pertence a um grupo de risco - 44\%; supostos eventos adversos da vacina $19 \%$; crença na ineficácia da vacina - 13\%

16 Baixo nível educacional relacionou-se a menores índices de vacinação

19 Crença de que não pertence a grupos de risco - 40,6\%; não ter vacinação prévia - 34,5\%; a gripe não se constituir em doença de severidade - 45,6\%; medo de agulhas - 5,6\%

24 Crença, por parte do trabalhador da saúde, de que existem poucos estudos que comprovem a efetividade da vacina - 75,7\%; posição pessoal contra a vacina - 55,9\%; percepção de que a gripe por H1N1 é uma doença benigna - 51,5\%

25 Eventos adversos relacionados ao ovo ou a outros componentes da vacina; crença que a vacina pode causar doença

27 Falta de tempo - 35,9\%; percepção de que estão fora do grupo de risco - 25\%; percepção de que não precisam da vacina porque não têm contato com pacientes de risco - 10,9\%

Alta confiança na sua condição imunológica; a vacinação não é necessária; desconfiança na segurança da vacina

30 Não apresentaram motivos para não vacinarem-se - 43\%; tinham receio dos eventos adversos - 18\%; sentiram-se doentes em vacinação prévia - 16\%

Não ter a vacina à disposição - 43\%; medo de contrair a doença - 16\%; acreditar que não estavam sob risco, porque eram jovens e saudáveis - 13\%; desconhecer a disponibilidade da vacina no serviço - $13 \%$

34 Medo dos supostos eventos adversos - 31,1\%; desconhecimento da severidade da doença $20,5 \%$; acreditar que o profissional não está sob maior risco de ter a doença - $8,3 \%$

A vacina não foi ofertada - 59,2\%; acreditar que não pertence a um grupo de risco específico para gripe, bem como as suas complicações - 40,8\%

39 Desconfiança e lapso no conhecimento da efetividade da vacina

41 Considerar que a gripe é uma doença leve - 36,9\%; baixa eficácia da vacina - 28,8\%

42 Ineficácia da vacina - 37,1\%; mal-estar provocado pela vacina - 21\%; ter baixo risco de infectar-se - $20 \%$

48 Desconfiar da efetividade da vacina

49 Lacunas no conhecimento sobre gripe e sobre a vacinação contra influenza

$51 \quad$ Baixo acesso à vacina; preconceitos contra a vacina

53 Crença de que companhias farmacêuticas influenciam decisões sobre estratégias de vacinação; discordar da vacinação - 34,5\%; a proteção conferida depende de fatores mais consistentes - 30,8\%; proteção e eficácia da vacina não é de $100 \%-22,7 \%$ 
$54 \quad$ Falta de tempo; desconhecer que a vacina é recomendada para os profissionais e estudantes da área de saúde

58 Não acreditar na vacina; não considerar a gripe como doença grave; pensar que não vai adoecer; ser pessoalmente contra a vacina

Apesar de os estudos incluídos na presente revisão terem sido realizados em diferentes ambientes de atenção à saúde e avaliarem variadas categorias profissionais bem como estudantes, pôde-se ter um quadro das taxas de vacinação em diversos tipos de serviços e em vários países. Identificaram-se também os fatores mais recorrentes que contribuíram para aumentar ou diminuir a adesão dos profissionais e estudantes da área da saúde à vacinação contra a gripe.

As percentagens de aderência à vacina oscilaram de 5,3\% a $94 \%$. Esses resultados desvelam que mesmo com a comprovada eficácia da vacina na proteção dos profissionais da saúde e, em consequência, também de seus familiares e, especialmente, dos pacientes sob os seus cuidados, ainda assim se apresentam disparidades na adesão, permanecendo, na média, abaixo do ideal.

Parece haver, todavia, conforme já mencionado, alguma confluência dos dados quando são cotejados por países. Isso nos permite supor que as taxas de adesão à vacinação possam depender mais das políticas governamentais sobre a imunização dos profissionais da saúde do que exatamente de fatores relacionados especificamente aos profissionais.

Há, porém, indicativos de que ocorre também a interferência de variáveis de formação profissional na aderência à vacina. E, além delas, estão presentes, de igual modo, outros aspectos relacionados à aceitação da vacina contra a gripe, para além da categoria profissional.

Em duas pesquisas aparece o sexo masculino como preditivo de maior adesão à vacina ${ }^{28,62}$. A idade dos profissionais também consta como dessemelhança na aderência à vacina. Encontrou-se a idade acima de 40 anos como preditivo de maior adesão ao imunobiológico ${ }^{15,26,28}$. Um estudo malaio, todavia, publicado mais recentemente, chegou a melhores resultados de vacinação nas faixas etárias mais baixas ${ }^{64}$. Por outro lado, em trabalho realizado com enfermeiras e ocupacionais de enfermagem atuantes em serviços domiciliares nos Estados Unidos, verificou-se que as trabalhadoras jovens e negras foram menos aderentes à vacina e que ser graduada em enfermagem foi relacionada a maiores taxas de vacinação, quando comparadas àquelas que não o eram. Detectou-se, adicionalmente, que as enfermeiras brancas demonstraram acreditar mais na eficácia do imunobiológico ${ }^{46}$.

De outra parte, nesse mesmo estudo, ficou demonstrado que as percentagens de vacinação foram $12 \%$ maiores em instituições que dispunham de dispositivos de incentivo à vacinação ${ }^{46}$. Mencionem-se, ainda, que a formação acadêmica foi elencada como fator positivo à aderência à vacinação, ou seja, quanto mais altos os níveis educacionais, maiores os índices de adesões à vacina ${ }^{26,52}$.

Por sua vez, os resultados das pesquisas com estudantes apontam para a necessidade de melhor atenção na sua formação e também na existência de políticas mais claras de vacinação dessa categoria. Se, por um lado, os estudantes mantêm contato com pacientes colocando-se em risco de adquirir gripe e também de transmitir para eles a infecção, por outro, as políticas institucionais, quando existem, parecem ser muito mais direcionadas a seus trabalhadores.

Note-se que uma pesquisa realizada com estudantes de medicina mostrou como preditores de adesão à vacina morar no próprio campus, ter experiência clínica e concordar que a vacina é importante ${ }^{60}$. Outra pesquisa que também revela resultados positivos em se tratando de alunos da área da saúde, mostrou que a aderência à vacinação foi estatisticamente relacionada ao conhecimento correto sobre a vacina e com a leitura de artigos científicos sobre o assunto ${ }^{14}$.

Corroboram com essas constatações a assertiva de que as instituições de ensino na área da saúde têm papel primordial na prevenção e controle das doenças imunopreviníveis, uma vez que durante a formação acadêmica se fundamentam conceitos e valores.

Assim, cabe a observação de que não 
obstante motivos pessoais atuem na decisão de tomar ou não a vacina, as políticas institucionais de recomendar fortemente a vacinação dos profissionais da saúde, de ofertar gratuitamente a vacina aos trabalhadores e, ainda, contribuir com a formação em serviço dos profissionais, têm relevância indubitável.

Essa consideração vem ao encontro dos resultados apresentados no artigo australiano que exibiu um incremento na taxa de vacinação, passando de $76 \%$ para $80 \%$, após a realização de campanha de conscientização. São citados, como elementos que concorreram para uma maior aderência, a existência da vacina à disposição e os trabalhos de comunicação e marketing ${ }^{52}$.

Imperativo dar relevo às investigações científicas realizadas pelo CDC, nos Estados Unidos, que detectaram maiores taxas de adesão à vacina quando esta é oferecida gratuitamente, no próprio local de trabalho e, mais que isso, embora objeto de controvérsias, quando a vacinação se constitui em demanda obrigatória nos serviços ${ }^{67}$.

Importante salientar que em vários estudos aqui analisados cita-se a subestimação do risco de se adquirir gripe como elemento que afasta os profissionais e também estudantes de se vacinarem.

A gripe é, com frequência, considerada como de pouca gravidade e não se dá a devida importância ao risco da veiculação do vírus aos pacientes.

Naqueles estudos em que se fazem as gradações das principais razões dos profissionais e estudantes de se vacinarem, quase sempre a proteção dos pacientes aparece, em que pese sua importância, quando muito, a partir do segundo ou terceiro lugar ${ }^{11,13,24,38,47}$.

\section{Conclusão}

A vacinação antigripal tem sido defendida como importante elemento na proteção dos profissionais de saúde, com implicações positivas para esses trabalhadores, redução no absenteísmo ao trabalho e consequente diminuição dos custos financeiros nos serviços. Destaca-se, especialmente, a importância da vacina na diminuição do risco dos profissionais e estudantes de contaminar os pacientes sob os seus cuidados.

Todavia, os autores são unânimes em evidenciar que há grande variação nas taxas de adesão à vacina anti-influenza e que essa aderência é quase sempre aquém do desejável.

Comprova-se, com a presente revisão, que, de fato, há variabilidade substantiva nas taxas de vacinação e que também elas se apresentam, na maioria das vezes, inferiores ao necessário.

Entretanto, cumpre mencionar que pela análise dos artigos pôde-se vislumbrar certa recorrência nos resultados quando esses são tomados por países. Isso permite ponderar que a vacinação dos profissionais parece depender mais das políticas governamentais para 0 gerenciamento do assunto do que exclusivamente de razões pessoais dos trabalhadores. A oferta gratuita da vacina, preferencialmente no próprio local de trabalho, e até a obrigatoriedade da vacinação, afigura-se como um exemplo.

Importa salientar, no entanto, as naturais limitações do estudo para uma afirmação categórica nesse sentido. O número de trabalhos analisados, por país, foi modesto e, assim, eles não refletem, necessariamente, os resultados de todas as suas regiões e serviços.

Para se confirmar ou se refutar a possiblidade aqui colocada, outros estudos, mais verticais, por país, utilizando uma mesma sistemática e metodologia, a exemplo até do que vem sendo realizado por um ou outro grupo de pesquisadores, deveriam ser realizados.

Também, a estrutura organizacional das instituições parece ter relevância na adesão à vacina. Alguns estudos aqui analisados tratam, de algum modo, desse assunto, mas não se constitui na temática principal das pesquisas. Parte substantiva dos trabalhos centram-se nos motivos pessoais que levam o indivíduo a se vacinar ou a sua recusa à vacina.

Assim, pensamos que complementarmente às pesquisas com a população de profissionais de saúde e estudantes da área, devam ser analisadas outras investigações científicas, com ênfase maior nas instituições e nos seus mecanismos de controle da vacinação.

\section{Referências}

1. Salgado CD, Farr BM, Hall KK, Hayden FG. Influenza in the acute hospital setting. Lancet Infect Dis [Internet]. 2002;2(3):145-55. Available at:

http://www.sciencedirect.com/science/article/pii /S1473309902002219

2. Trombetta C, Piccirella S, Perini D, Kistner O, Montomoli E. Emerging Influenza Strains in the Last Two Decades: A Threat of a New Pandemic? Vaccines [Internet]. 2015;3(4):172-85. Available at: http://www.mdpi.com/2076-393X/3/1/172

3. Jones RM, Xia Y. Annual Burden of Occupationally-Acquired Influenza Infections in 
Hospitals and Emergency Departments in the United States. Risk Anal. 2018;38(3):442-53.

4. Kuster SP, Shah PS, Coleman BL, Lam PP, Tong $A$, Wormsbecker $A$, et al. Incidence of influenza in healthy adults and healthcare workers: A systematic review and meta-analysis. PLoS One. 2011;6(10):1-9.

5. Elder AG, Symington IS, O'donnell B, Mccruden EAB, Carman WF. Incidence and recall of influenza in a cohort of Glasgow healthcare workers during the 1993-4 epidemic: Results of serum testing and questionnaire. Bmj. 1996;313(7067):1241-2.

6. Gianino MM, Politano G, Scarmozzino A, Charrier L, Testa M, Giacomelli S, et al. Estimation of sickness absenteeism among Italian healthcare workers during seasonal influenza epidemics. PLoS One. 2017;12(8):e0182510.

7. Who. Vaccines against influenza WHO position paper - November 2012. Wkly Epidemiol Rec. 2012;47(87):461-76.

8. European Centre for Disease Prevention and Control (ECDC). ECDC Technical Report: Seasonal influenza vaccination in Europe [Internet]. 2017. 33 p. Available at: https://ecdc.europa.eu/sites/portal/files/docum ents/influenza-vaccination-2007?2008-to2014?2015.pdf

9. Acip IP. Immunization of Health-Care Personnel Recommendations of the Advisory Committee on. Contin Educ [Internet]. 2011;60(7):1-45. Available at: http://www.ncbi.nlm.nih.gov/pubmed/22108587 10. Savio E, Celi AP, Sartori GP, Vásquez H. Vacunaciones de los adultos: Manual práctico. $3^{\circ}$ ed. Quito: Asociación Panamericana de Infectologia; 2017. 346 p.

11. Wicker S, Rabenau HF, Betz W, Lauer HC. Attitudes of dental healthcare workers towards the influenza vaccination. Int J Hyg Environ Health [Internet]. 2012;215(4):482-6. Available at: http://dx.doi.org/10.1016/j.ijheh.2011.08.005

12. Landelle $C$, Vanhems $P$, Saadatian-Elahi $\mathrm{M}$, Voirin $\mathrm{N}$. Influenza vaccination coverage among patients and healthcare workers in a university hospital during the 2006-2007 influenza season. Vaccine [Internet]. 2012;31(1):23-6. Available at: http://dx.doi.org/10.1016/j.vaccine.2012.10.059

13. Chen SC, Hawkins G, Aspinall E, Patel N. Factors influencing uptake of influenza A (H1N1) vaccine amongst healthcare workers in a regional pediatric centre: Lessons for improving vaccination rates. Vaccine [Internet]. 2012;30(2):493-7. Available at: http://dx.doi.org/10.1016/j.vaccine.2011.04.032 14. Lee SI, Aung EM, Chin IS, Hing JW,
Mummadi S, Palaniandy GD, et al. Factors Affecting Medical Students' Uptake of the 2009 Pandemic Influenza A (H1N1) Vaccine. Influenza Res Treat [Internet]. 2012;2012:753164. Available at: http://www.pubmedcentral.nih.gov/articlerende r.fcgi?artid=3515892\&tool=pmcentrez\&renderty pe=abstract

15. Bouadma L, Barbier F, Biard L, EspositoFarese M, Le Corre B, Macrez A, et al. Personal Decision-Making Criteria Related to Seasonal and Pandemic A (H1N1) Influenza-Vaccionation Acceptance among Healthcare Workers. PLoS One. 2012;7(7):e38646.

16. Böhmer MM, Walter D, Falkenhorst G, Müters S, Krause G, Wichmann O. Barriers to pandemic influenza vaccination and uptake of seasonal influenza vaccine in the post-pandemic season in Germany. BMC Public Health. 2012;12(1).

17. Sawyer $\mathrm{MH}$, Peddecord KM, Wang $\mathrm{W}$, Deguire $M$, Miskewitch-Dzulynsky $M$, Vuong DD. A public health initiative to increase annual influenza immunization among hospital health care personnel: The San Diego Hospital Influenza Immunization Partnership. Am J Infect Control [Internet]. 2012;40(7):595-600. Available at: http://dx.doi.org/10.1016/j.ajic.2011.09.007

18. Honda $\mathrm{H}$, Padival $\mathrm{S}$, Shimamura $\mathrm{Y}$, Babcock HM. Changes in influenza vaccination rates among healthcare workers following a pandemic influenza year at a Japanese tertiary care centre. J Hosp Infect [Internet]. 2012;80(4):316-20. Available at: http://dx.doi.org/10.1016/j.jhin.2011.12.014

19. Lu PJ, Ding H, Black CL. H1N1 and seasonal influenza vaccination of U.S. healthcare personnel, 2010. Am J Prev Med. 2012;43(3):282-92.

20. Costa JT, Silva R, Tavares M, Nienhaus A. High effectiveness of pandemic influenza $A$ (H1N1) vaccination in healthcare workers from a Portuguese hospital. Int Arch Occup Environ Health. 2012;85(7):747-52.

21. Sánchez-Payá J, Hernández-García I, García-Román V, Camargo-Angeles R, Barrenengoa-Sañudo J, Villanueva-Ruiz $\mathrm{CO}$, et al. Influenza vaccination among healthcare personnel after pandemic influenza H1N1. Vaccine. 2012;30(5):911-5.

22. Ball S, Walker D, Donahue SM, Izrael D, Zhang J, Euler GL, et al. Influenza Vaccination Coverage Among Health-Care Personnel - 201112 Influenza Season, United States. mor [Internet]. 2012;61(38):768-71. Available at: http://www.ncbi.nlm.nih.gov/pubmed/23013723

23. Rebmann T, Wright KS, Anthony J, Knaup 
RC, Peters EB. Seasonal and H1N1 influenza vaccine compliance and intent to be vaccinated among emergency medical services personnel. Am J Infect Control [Internet]. 2012;40(7):632-6. Available http://dx.doi.org/10.1016/j.ajic.2011.12.016

24. Guevara M, Toledo D, Zabala A, Castilla J. Actitudes, percepciones y factores asociados a la vacunación antigripal en los profesionales de atención primaria de Navarra , 2011-2012 Attitudes, perceptions and factors associated with influenza. 2013;36:2011-2.

25. Zhou L, Su Q, Xu Z, Feng A, Jin H, Wang S, et al. Seasonal Influenza Vaccination Coverage Rate of Target Groups in Selected Cities and Provinces in China by Season (2009/10 to 2011/12). PLoS One. 2013;8(9):1-7.

26. Barbadoro P, Marigliano A, Di Tondo E, Chiatti C, Di Stanislao F, D'Errico MM, et al. Determinants of influenza vaccination uptake among Italian healthcare workers. Hum Vaccines Immunother. 2013;9(4):911-6.

27. Civljak R, Papic N, Stamenic V, Kalenic S, Kuzman I, Car J. Influenza and hepatitis B vaccination coverage among healthcare workers in Croatian hospitals: A series of cross-sectional surveys, 2006-2011. BMC Infect Dis. 2013;13(1):1-8.

28. Domínguez A, Godoy P, Castilla J, Soldevila N, Toledo D, Astray J, et al. Knowledge of and attitudes to influenza vaccination in healthy primary healthcare workers in Spain, 2011-2012. PLoS One. 2013;8(11):2011-2.

29. Bonaccorsi G, Lorini C, Santomauro F, Guarducci S, Pellegrino E, Puggelli F, et al. Predictive factors associated with the acceptance of pandemic and seasonal influenza vaccination in health care workers and students in Tuscany, Central Italy. Hum Vaccines Immunother. 2013;9(12):2603-12.

30. Llupià A, Mena G, Olivé V, Quesada S, Aldea $M$, Sequera VG, et al. Evaluating influenza vaccination campaigns beyond coverage: A before-after study among health care workers. Am J Infect Control [Internet]. 2013;41(8):674-8. Available http://dx.doi.org/10.1016/j.ajic.2013.04.006

31. Person CJ, Nadeau JA, Schaffzin JK, Pollock L, Wallace BJ, McNutt LA, et al. Influenza immunization coverage of residents and employees of long-term care facilities in New York State, 2000-2010. Am J Infect Control [Internet]. 2013;41(8):743-5. Available at: http://dx.doi.org/10.1016/j.ajic.2012.09.025

32. Lee SJ, Harrison R, Rosenberg J, McLendon $\mathrm{P}$, Boston $\mathrm{E}$, Lindley MC. Influenza vaccination among health care personnel in
California: 2010-2011 influenza season. Am J Infect Control [Internet]. agosto de 2013;41(8):e65-71. Available at: http://linkinghub.elsevier.com/retrieve/pii/S019 6655312013430

33. Ball SW, Donahue SM, Izrael D, Walker D, DiSogra C, Martonik $\mathrm{R}$, et al. Vaccination Coverage Among Health-Care Personnel United States , 2012 - 13 Influenza Season. Morb Mortal Wkly Rep. 2013;62(38):781-6.

34. Valour F, Bénet $T$, Chidiac C. Pandemic $A(H 1 N 1) 2009$ influenza vaccination in Lyon University Hospitals, France: Perception and attitudes of hospital workers. Vaccine. 2013;31(4):592-5.

35. Corace K, Prematunge C, McCarthy A, Nair RC, Roth V, Hayes $T$, et al. Predicting influenza vaccination uptake among health care workers: What are the key motivators? Am J Infect Control [Internet]. 2013;41(8):679-84. Available http://dx.doi.org/10.1016/j.ajic.2013.01.014

36. Crowley KA, Myers R, Magda LA, Morse SS, Brandt-Rauf $P$, Gershon RRM. Prevalence and factors associated with 2009 to 2011 influenza vaccinations at a university medical center. Am J Infect Control [Internet]. 2013;41(9):824-30. Available http://dx.doi.org/10.1016/j.ajic.2012.11.020

37. Afonso $\mathrm{N}$, Kavanagh $\mathrm{M}$, Swanberg $\mathrm{S}$. Improvement in attitudes toward influenza vaccination in medical students following an integrated curricular intervention. Vaccine [Internet]. 2014;32(4):502-6. Available at: http://dx.doi.org/10.1016/j.vaccine.2013.11.043

38. Prematunge $\mathrm{C}$, Corace $\mathrm{K}$, McCarthy A, Nair RC, Roth V, Suh KN, et al. Qualitative motivators and barriers to pandemic vs. seasonal influenza vaccination among healthcare workers: A content analysis. Vaccine [Internet]. 2014;32(52):7128-34. Available at: http://dx.doi.org/10.1016/j.vaccine.2014.10.023

39. Garcell HG, Ramirez EC. Influenza immunization coverage for healthcare workers in a community hospital in Qatar (2011-2012 and 2012-2013 seasons). J Infect Public Health [Internet]. 2014;7(1):70-2. Available at: http://dx.doi.org/10.1016/j.jiph.2013.06.007

40. Jaiyeoba O, Villers M, Soper DE, Korte J, Salgado CD. Association between health care workers' knowledge of influenza vaccine and vaccine uptake. Am J Infect Control [Internet]. 2014;42(1):69-70. Available at: http://dx.doi.org/10.1016/j.ajic.2013.06.020

41. Naleway AL, Henkle EM, Ball S, Bozeman S, Gaglani MJ, Kennedy ED, et al. Barriers and facilitators to influenza vaccination and vaccine 
coverage in a cohort of health care personnel. Am J Infect Control [Internet]. 2014;42(4):371-5. Available

at: http://dx.doi.org/10.1016/j.ajic.2013.11.003

42. Alshammari TM, AlFehaid LS, AlFraih JK, Aljadhey HS. Health care professionals' awareness of, knowledge about and attitude to influenza vaccination. Vaccine [Internet]. 2014;32(45):5957-61. Available at: http://dx.doi.org/10.1016/j.vaccine.2014.08.061 43. Black $C L$, Yue $X$, Ball SW, Donahue SM, Izrael D, Perio MA, et al. Influenza Vaccination Coverage Among Health Care Personnel United States, 2013-14 Influenza Season. MMWR Morb Mortal Wkly Rep [Internet]. 2014;63(37):812-5. Available at: http://www.ncbi.nlm.nih.gov/pubmed/25233282 44. Albano L, Matuozzo A, Marinelli P, Di Giuseppe G. Knowledge, attitudes and behaviour of hospital health-care workers regarding influenza A/H1N1: A cross sectional survey. BMC Infect Dis [Internet]. 2014;14(1):1-7. Available at: BMC Infectious Diseases

45. Bentele $\mathrm{H}$, Bergsaker MR, Hauge $\mathrm{SH}$, Bjørnholt J V. Vaccination coverage for seasonal influenza among residents and health care workers in Norwegian nursing homes during the 2012/13 season, a cross-sectional study. BMC Public Health. 2014;14(1):2-7.

46. Daugherty JD, Blake SC, Grosholz JM, Omer SB, Polivka-West L, Howard DH. Influenza vaccination rates and beliefs about vaccination among nursing home employees. Am J Infect Control [Internet]. 2015;43(2):100-6. Available at: http://dx.doi.org/10.1016/j.ajic.2014.08.021

47. Hernández-García I, Cardoso-Muñoz AM, Valero-Juan LF, Giménez-Júlvez MT. Vacunación antigripal en estudiantes de enfermería durante la temporada 2014-2015. Rev Esp Salud Pública. 2015;89:615-25.

48. Montserrat-Capdevila J, Godoy P, Marsal JR, Barbé-Illa F. Factores asociados a recibir la vacunación antigripal en profesionales de atención primaria TT - Factors associated with influenza immunization in primary care health workers. Gac Sanit [Internet]. 2015;29(5):383-6. Available at: http://scielo.isciii.es/scielo.php?script=sci_arttex t\&pid=S0213-

91112015000500013\&lang=enhttp://scielo.isciii. es/pdf/gs/v29n5/original_breve4.pdfhttp://www. scielosp.org/scielo.php?script=sci_arttext\&pid=S 0213-91112015000500013\&lang=en

49. Lehmann BA, Ruiter RAC, van Dam D, Wicker S, Kok G. Sociocognitive predictors of the intention of healthcare workers to receive the influenza vaccine in Belgian, Dutch and German hospital settings. J Hosp Infect [Internet]. 2015;89(3):202-9. Available at: http://dx.doi.org/10.1016/j.jhin.2014.11.009

50. Antón-Ladislao A, García-Gutiérrez S, Soldevila N, González-Candelas F, Godoy P, Castilla $J$, et al. Visualizing knowledge and attitude factors related to influenza vaccination of physicians. Vaccine. 2015;33(7):885-91.

51. Cozza V, Alfonsi V, Rota MC, Paolini V, Ciofi Degli Atti ML. Promotion of influenza vaccination among health care workers: Findings from a tertiary care children's hospital in Italy. BMC Public Health [Internet]. 2015;15(1):1-7. Available at: http://dx.doi.org/10.1186/s12889015-2067-9

52. Heinrich-Morrison $\mathrm{K}$, McLellan $\mathrm{S}$, McGinnes U, Carroll B, Watson K, Bass P, et al. An effective strategy for influenza vaccination of healthcare workers in Australia: Experience at a large health service without a mandatory policy. BMC Infect Dis. 2015;15(1):1-8.

53. Chambers LW, Crowe L, Lam P-P, MacDougall D, McNeil S, Roth V, et al. A New Approach to Improving Healthcare Personnel Influenza Immunization Programs: A Randomized Controlled Trial. PLoS One [Internet]. 2015;10(3):e0118368. Available at: http://dx.plos.org/10.1371/journal.pone.011836 8

54. Tuckerman JL, Collins JE, Marshall HS. Factors affecting uptake of recommended immunizations among health care workers in South Australia. Hum Vaccin Immunother [Internet]. 2015;11(3):704-12. Available at: http://www.tandfonline.com/doi/full/10.1080/2 1645515.2015.1008886

55. Alicino C, ludici R, Barberis I, Paganino C, Cacciani $R$, Zacconi $M$, et al. Influenza vaccination among healthcare workers in Italy. Hum Vaccin Immunother [Internet]. 2015;11(1):95-100. Available at: http://www.pubmedcentral.nih.gov/articlerende r.fcgi?artid $=4514208 \&$ tool=pmcentrez\&renderty pe=abstract

56. Edge R, Heath J, Rowlingson B, Keegan TJ, Isba R. Seasonal influenza vaccination amongst medical students: A social network analysis based on a cross-sectional study. PLoS One. 2015;10(10):1-13.

57. Giannattasio A, Mariano M, Romano R, Chiatto F, Liguoro I, Borgia G, et al. Sustained low influenza vaccination in health care workers after H1N1 pandemic: A cross sectional study in an Italian health care setting for at-risk patients. BMC Infect Dis [Internet]. 2015;15(1):1-8. Available at: http://dx.doi.org/10.1186/s12879015-1090-x 
58. Kadi Z, Atif ML, Brenet A, Izoard S, Astagneau $P$. Barriers of influenza vaccination in health care personnel in France. Am J Infect Control [Internet]. 2016;44(3):361-2. Available at http://dx.doi.org/10.1016/j.ajic.2015.09.027

59. Costantino C, Amodio E, Calamusa G, Vitale F, Mazzucco W. Could university training and a proactive attitude of coworkers be associated with influenza vaccination compliance? A multicentre survey among Italian medical residents Assessment and evaluation of admissions, knowledge, skills and attitudes. BMC Med Educ [Internet]. 2016;16(1):1-6. Available at: http://dx.doi.org/10.1186/s12909-016-0558-8

60. Walker L, Newall A, Heywood AE. Knowledge, attitudes and practices of Australian medical students towards influenza vaccination. Vaccine [Internet]. 2016;34(50):6193-9. Available at:

http://dx.doi.org/10.1016/j.vaccine.2016.10.074

61. Asma $S$, Akan $H$, Uysal $Y$, Poçan AG, Sucakli $\mathrm{MH}$, Yengil $\mathrm{E}$, et al. Factors effecting influenza vaccination uptake among health care workers: A multi-center cross-sectional study. BMC Infect Dis. 2016;16(1):1-9.

62. Durando P, Alicino C, Dini G, Barberis I, Bagnasco AM, ludici $R$, et al. Determinants of adherence to seasonal influenza vaccination among healthcare workers from an Italian region: Results from a cross-sectional study. BMJ Open. 2016;6(5):1-9.

63. Ivani De Paula S, Ivani De Paula G, Simone K. Adherence To Influenza Vaccination Among Medical Students During and After Influenza a (H1N1) Pandemic. Rev Inst Med Trop Sao Paulo [Internet]. 2016;58. Available at: http://dx.doi.org/10.1590/S1678-

9946201658082

64. Hudu SA, Harmal NS, Malina O, Sekawi Z. Influenza vaccination among Malaysian healthcare workers: A survey of coverage and attitudes. Med J Malaysia. 2016;71(5):231-7.

65. Black $C L$, Yue $X$, Ball SW, Donahue SMA, Izrael $D$, de Perio MA, et al. Influenza Vaccination Coverage Among Health Care Personnel United States, 2015-16 Influenza Season. Mmwr [Internet]. 2016;65(38):1026-31. Available at: http://www.cdc.gov/mmwr/volumes/65/wr/mm 6538a2.htm

66. Assaf AM, Hammad EA, Haddadin RN. Influenza Vaccination Coverage Rates, Knowledge, Attitudes, and Beliefs in Jordan: A Comprehensive Study. Viral Immunol [Internet]. 2016;29(9):516-25. Available at: http://online.liebertpub.com/doi/10.1089/vim.2 015.0135
67. Conte A, Quattrin R, Filiputti E, Cocconi R, Arnoldo $L$, Tricarico $P$, et al. Promotion of flu vaccination among healthcare workers in an Italian academic hospital: An experience with tailored web tools. Hum Vaccin Immunother [Internet]. 2016;12(10):2628-33. Available at: https://www.tandfonline.com/doi/full/10.1080/2 1645515.2016.1186319

\section{Endereço para Correspondência}

Universidade Estadual de Santa Cruz - UESC

Campus Soane Nazaré de Andrade, Rod. Jorge

Amado, Km 16 - Salobrinho, Ilhéus - BA

CEP.: 45662-900

e-mail: Imskalinski@yahoo.com.br

Recebido em 12/11/2018

Aprovado em 18/09/2019

Publicado em 13/02/2020 